\title{
Online Ship Rolling Estimation Using a Grey Support Vector Machine Prediction Scheme
}

\section{Cheng Liu ${ }^{1}$}

Navigation College,Dalian Maritime University, Dalian, 116026, China

E-mail: Ic5355010640163.com

Jian-chuan Yin ${ }^{2}$

Navigation College, Dalian Maritime University, Dalian,116026, China

E-mail: yinjianchuanedlmu.edu.cn

\section{Xin-guang Zhang ${ }^{3}$}

Automobile Engineering College, Shang Hai University of engineering Science, Shanghai,200000, China E-mail:535501064@qq. com

\section{Xue-gang Wang}

Fourth Harbor Engineering Institute Co., Ltd., China Communication Construction Company,

Guangdong, 510000, China

E-mail: $773122640 @ q q$. com

\begin{abstract}
An online sequential grey predictive scheme is proposed by embedding the supporting vector machine (SVM) in a grey prediction framework. The grey processing of time series alleviates the unfavorable effects resulted from uncertainty available in the measurement data and the nonlinear and self-adaptation natures of SVM enable accurate approximation of the scheme. The resulted grey SVM predictor can be utilized to represent the nonlinear mapping influenced by uncertainty. The Ship's motion at sea is affected by various time-varying environmental factors. As a result, the ship's rolling motion is a complex nonlinear system which is hard to be predicted precisely by custom approaches. In this paper, the grey SVM predictor is utilized for online ship rolling angle prediction. The prediction simulation is performed based on the measurement data from scientific research and training ship Yu Kun. Simulation results have demonstrated that the proposed method can give predictions for ship rolling motion in real time with high accuracy and satisfactory stability.
\end{abstract}

CENet2015

12-13 September 2015

Shanghai, China

\footnotetext{
${ }^{1}$ Speaker

${ }^{2}$ Corresponding Author

${ }^{3}$ Acknowledgment: this work is supported by National Nature Science Foundation of China (Grant No.: 51279106), the Natural Science Foundation of Liaoning Province (Grant No.: 201425008), the General Project of Liaoning Province (Grant No. L2014214) and the Fundamental Research Funds for the Central Universities of China (Grant No.: 3132014028, 3132014309).
} 


\section{Introduction}

The Ship's motion at sea is a complex system whose dynamics is related to the sailing conditions such as trim, draft and loading condition. It is also inevitably disturbed by the environmental conditions such as wind, wave and current [1]. The dynamics of ship rolling is featured by strong nonlinearity, time varying and high randomness. As stated above, it is difficult to identify and predict the rolling motion with conventional linear methods [2].

The newly-developed artificial intelligent computation techniques, such as neural networks, fuzzy logic inference and evolutionary computation, have drawn the attention of researchers in the marine field. $\mathrm{Xu}$ et al. created an online modeling of ship maneuvering motion based on SVM [3].

Grey system theory is a generic theory in processing system whose information involves uncertain or incomplete meanings [4]. Nowadays, it has been improved in combination with intelligent computational techniques such as genetic algorithms $[5,6]$, fuzzy systems and neural networks[7,8].

In this paper, the grey prediction model is employed to process raw data so as to abate unfavorable effects of uncertainty. SVM is utilized to realize the nonlinear mapping of grey system. The combination of the two models can not only predict the ship rolling angle with high accuracy but also eliminate some random disturbance. As the ship's rolling movement is a typical nonlinear system with uncertainty, the constructed modular predictive method is applied to the online ship rolling angle prediction. The simulation results demonstrate that the method has realized the nonlinear mapping within grey prediction scheme, which thus enables satisfactory accuracy and processing speed.

This paper is organized as follows. Section 2 provides the general introduction of SVM and grey prediction model, respectively. Section 3 gives a brief introduction to the proposed grey prediction model based on SVM. Section 4 gives the ship rolling motion and the result of the predictive model in the simulation. Finally the conclusion is made in section 5.

\section{SVM and Grey Prediction Model}

Support Vector Machine (SVM) was first scheduled in 1995 [9]. The main idea of SVM is to build up a classification hyperplane as the decision surface which makes the isolation edge maximized among positive example and counter example. The learning method of SVM is based on the statistical learning theory. SVM has many advantages as follows: (1) generality. SVM can construct function in extensively various kinds of function set; (2) robustness. SVM doesn't need to fine-tune its parameters; (3) simple calculation. The implementation of SVM only needs simple optimization technique; (4) complete theoretical system. SVM is constructed in VC patulous theory frame.

In the conventional time-series prediction approach, a large amount of data are usually needed for deriving predictions; however, the grey prediction approach has been demonstrated to be an effective method in the processing system with incomplete information with limited number of elements. It can provide input series effectively which is in a consecutive time order at equal intervals as well as the sample number should larger than four [10]. There are three fundamental operations in the grey prediction model: (1) accumulated generation operation (AGO) by accumulating the original series; (2) grey prediction based on the accumulated series in grey model; (3) inverse accumulated generation operation (IAGO) where the series in grey model is converted back to its origin to achieve the predicted value.

\section{Grey Prediction Model based on SVM}

Although AGO operation in grey prediction model can eliminate a lot of original uncertainties and noises caused by the measuring and indeterminacy factors, the processed data are still complex. In order to predict these complex data, SVM has its special superiority because of its inherent nonlinear nature. 
SVM has curve fitting ability for nonlinear mapping. Time series prediction method based on SVM can use historical data as input and produce output accordingly. Once the structure and parameters of SVM are determined by the processed data, the achieved SVM can be implemented to produce predictions.

In this study, the custom Autoregressive (AR) prediction model is implemented for prediction. That is, in the training process of $k$-th step, the input SVM are set as $x^{(1)}(k-1)$, $x^{(1)}(k-2) \ldots x^{(1)}(k-n)$, with the output set as $x^{(1)}(k)$, where $x^{(1)}(k-i)(i=0,1, \ldots, k)$ is the AGO results of the original series $x^{(0)}(k-i)(i=0,1, \ldots, k)$. The trained SVM network can be implemented for prediction immediately as follows:

$$
y=f\left(x^{(1)}(k-i)\right)
$$

Where $f$ is the nonlinear mapping realized by the trained SVM.

After the training process, the adjusted SVM network can be implemented for prediction. Upon prediction, the ultimate result $\hat{x}^{(0)}(k+1)$ should be achieved by implementing IAGO: $\hat{x}^{(0)}(k+1)=x^{(1)}(k+1)-x^{(1)}(k)$. The whole system is shown in Fig 1.

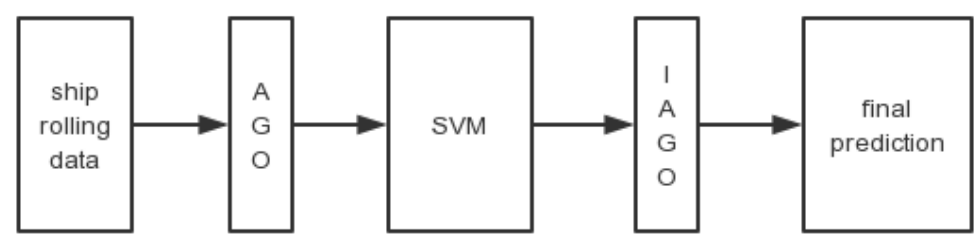

Figure 1: Data Processing Flowchart

It can be seen in Fig. 1 that, SVM is embedded in the framework of grey prediction thus construct a grey SVM. It also denotes the operations of accumulated generation operation and inverse accumulated generation operation in the grey prediction scheme. AGO operation is performed to the raw data to alleviate unfavorable effects of uncertainty and the processed data is conveyed to SVM for prediction. Upon prediction, the final prediction results are achieved by the IAGO operation.

\section{Online Ship Rolling Estimation Result and Discussion}

\subsection{Ship Rolling Motion}

In this paper, the Conolly equation is utilized as a typical ship rolling function. According to Conolly's equation, the ship rolling motion can be expressed as follows:

$$
I_{x x}^{\prime} \ddot{\varphi}+2 \mathrm{~N} \varphi+D h \varphi=D h a_{m} \sin \omega t
$$

Where $\varphi, \dot{\varphi}$ and $\ddot{\varphi}$ refer to the ship rolling angle, the ship's angular velocity and the acceleration of ship rolling angle, respectively. Here, $I^{\prime}{ }_{x x}$ represents the ship rolling inertia moment, $a_{m}$ is effective wave tilt, $\omega$ is the ratio of circumference of the waves, $h$ represents the vessel transverse metacentric height and $D$ is the ship's displacement.

By differential method, the prediction model can be derived. Firstly, $\varphi_{t}$ is represented by $f\left(\ddot{\varphi}_{t}\right)$. Based on differential formula $\ddot{\varphi}_{(t)}=\left(\left(\dot{\varphi}_{t}\right)-\left(\dot{\varphi}_{t-1}\right)\right) / \Delta t$, meanwhile $\dot{\varphi}_{t}$ and $\dot{\varphi}_{t-1}$ can be substituted as $\left(\left(\varphi_{t}\right)-\left(\varphi_{t-1}\right)\right) / \Delta t$ and $\left(\left(\varphi_{t-1}\right)-\left(\varphi_{t-2}\right)\right) / \Delta t$, respectively; thus the ship rolling angle at time $t$ can be represented according to the ship rolling angle at time $t-1$ and $t-2$. The function can be rewriten as a nonlinear form as follows:

$$
\varphi_{t}=f\left(\varphi_{t-1}, \varphi_{t-2}\right)
$$

The equation demonstrates that ship rolling angle can be predicted by ship rolling time series. 


\subsection{Simulation Result and Discussion}

In this section, all the data are come from the measurements of rolling angle on board $\mathrm{M}$. V. $Y U K U N$ during course-keeping sea trial at sea. $Y U K U N$ is a scientific research and training ship of Dalian Maritime University. The ship's movement information like heading angle, speed, position, roll, pitch and heave are measured and recorded by the ship movement status measurement unit automatically when the ship sails at sea. We choose a period of roll movement measurements for prediction purpose. The sampling time is $1 \mathrm{~s}$ and altogether 1500 rolling motion data are employed in this study. The measured roll angles show obvious nonlinear during the course-keeping process, as well as uncertainty to some extent. The orders of input and output are set as 2 and 1, respectively.

It is a one-step prediction, in the simulation, the first 1000 rolling data are willing to be an initial input to train the network for a sequence study process and the rest of data one by one without prediction result as input in order to simulate the test as an on-line situation.

The performance of a neural network is based on the structure and parameters of network, so it is important to choose SVM parameters such as the kind of kernel function, parameters of $c$ and $g$ and training epochs. An appropriate parameter may lead to a satisfactory performance. Inappropriate selection would lead to the phenomena of over-fitting or under-fitting. Under both conditions, the generalization performance of resulted network would be deteriorated.

To find the best parameter, the input data are calculated in the cross validation method and finally we choose $c$ as 20 and $g$ as 0.2 . With the selected parameters, the result of correlation coefficient (CC) is $99.98 \%$, which shows a strong correlation between prediction data with original data. The selection of kernel function is also very important. In the paper, four main kernels are tested in the simulation to choose the best one with the best performance. The results are shown in Table. 1.

\begin{tabular}{|l|l|l|l|}
\hline Kernel function & CC & RMSE $\left(^{\circ}\right)$ & Parameters \\
\hline Linear & 0.98 & $0.391^{\circ}$ & $-c 20-g 0.2-t 0$ \\
\hline Polynomial & 0.82 & $11.47^{\circ}$ & $-c 20-g 0.2-t 1$ \\
\hline Radial basis function & 0.99 & $0.385^{\circ}$ & $-c 20-g 0.2-t 2$ \\
\hline Sigmoid & 0.98 & $5.169^{\circ}$ & $-c 20-g 0.2-t 3$ \\
\hline
\end{tabular}

Table 1: Selection of Kernel Function

Where $t$ is the mark of four kernel function in procedure. RMSE is a custom evaluation indicator to measure the performance, the numerical value has better performance with the smaller RMSE value, which is shown in Equation (4.3).

$$
R M S E_{\text {pred }}=\sqrt{\frac{\sum_{i=1}^{L}(y-\hat{y})^{2}}{L}}
$$

Where $L$ is the total operation epochs; $y$ represents the real ship rolling value and $\hat{y}$ is the predicted value.

From Table 1, it is shown that the Polynomial and Sigmoid kernel function perform worse results in rolling prediction than others. The linear and radial basis function (RBF) ones have good performance and we choose RBF as the kernel function in this simulation which has high $\mathrm{CC}$ and less RMSE. Moreover, in this simulation, SVM is set as an $\varepsilon$ SVM because it is fit for regression and it has high accuracy which is an important index in prediction. The training epochs are set as 500 .

To compare the effect of prediction between the proposed grey SVM and the conventional SVM, both approaches are implemented for comparison. The result by using grey SVM is shown in Fig. 2 together with real rolling data. 


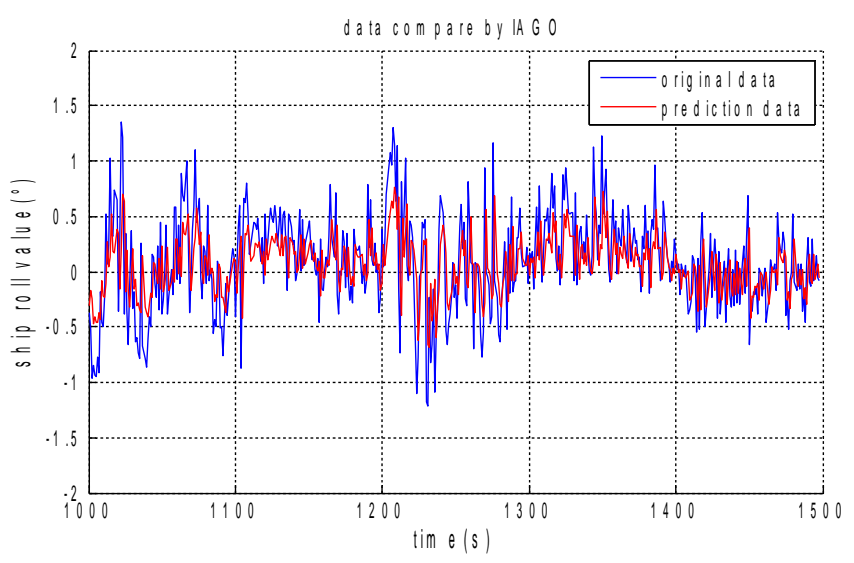

Figure 2: Final Result of Grey SVM Prediction by IAGO

It can be noticed in Fig. 2 that the Grey SVM can predict the holistic change of roll angle holistically. In order to depict the effect of prediction and analysis of the difference between two methods in a more clear manner, the prediction error of conventional SVM and grey SVM are shown in Fig. 3 and Fig. 4 respectively.

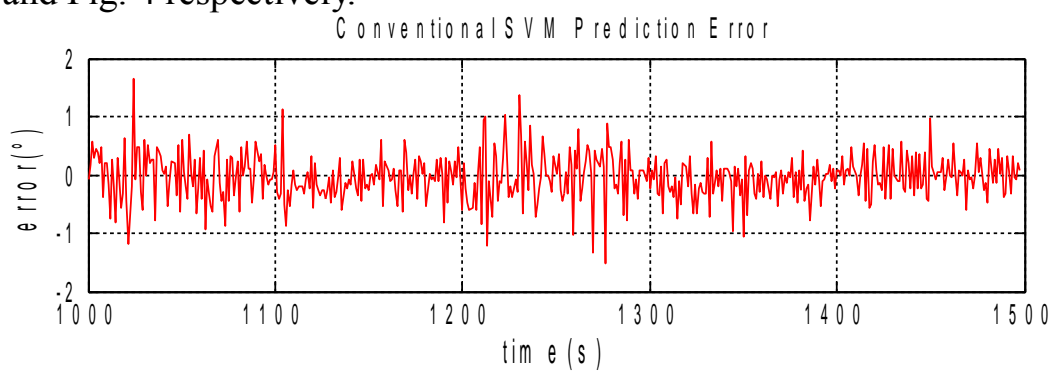

Figure 3: SVM Ship Prediction Error

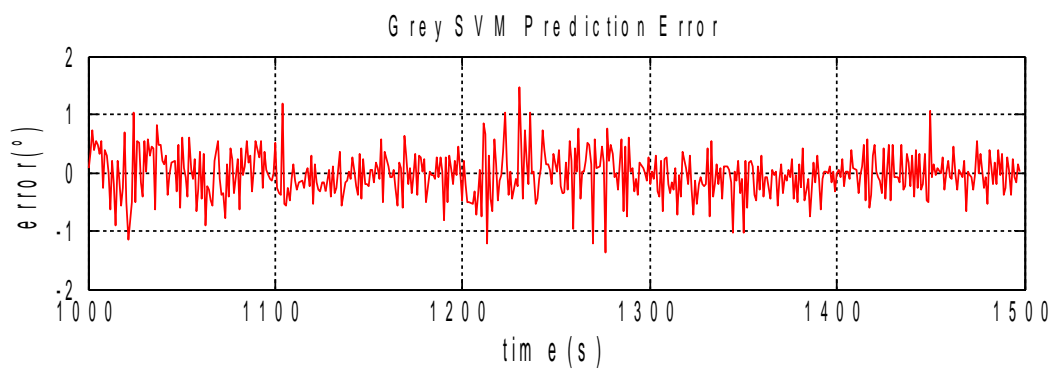

Figure 4 :Ship Prediction Error in Grey SVM

By comparing Fig. 3 and Fig. 4, it is noted that the error caused by conventional SVM shows a larger scale, constrained to $\pm 1.7^{\circ}$ and more severe changes; however, grey SVM error in Fig. 4 has better performance with smaller mean error value and the changes are more stable and concentrated.

In order to indicate the prediction error more clearly, we use RMSE to compare the purpose in the course of simulation. The prediction RMSEs of conventional SVM and proposed grey SVM network are $0.3952^{\circ}$ and $0.3858^{\circ}$ respectively.

It is shown that grey SVM has better accuracy than conventional SVM which can resort to the grey model in alleviate the effects of uncertainty. It is shown that the proposed predictive model can generate predictions with high accuracy by representing the nonlinear feature and alleviate the unfavorable effects by grey prediction method.

For the sake of further research, several other methods are used to predict the ship rolling with same data and similar simulation environment. The networks as we choose are BP and RBF network because BP is one of the most widely used neural networks with stable 
performance and broad applicability. In this simulation, we choose RBF as the kernel function which is same with RBF neural network but the original theory of these two networks are different, so the comparison between these two methods is to show which is better. Detailed simulation results are shown in Table 2.

\begin{tabular}{|l|l|l|l|l|l|}
\hline $\begin{array}{l}\text { Network } \\
\text { Type }\end{array}$ & $\mathbf{S S E}\left({ }^{\circ}\right)$ & MSE $\left({ }^{\circ}\right)$ & MAE $\left({ }^{\circ}\right)$ & RMSE $\left({ }^{\circ}\right)$ & $\begin{array}{l}\text { Prediction } \\
\text { Time(s) }\end{array}$ \\
\hline SVM & 78.09 & 0.1562 & 0.3050 & 0.3952 & 0.0092 \\
\hline SVM-Grey & 74.42 & 0.1488 & 0.3000 & 0.3858 & 0.0104 \\
\hline BP & 93.70 & 0.1874 & 0.3320 & 0.4329 & 0.037 \\
\hline BP-Grey & 89.55 & 0.1790 & 0.3263 & 0.4232 & 0.044 \\
\hline RBF & 146.99 & 0.2940 & 0.3354 & 0.5422 & 0.062 \\
\hline RBF-Grey & $2.0 \mathrm{e}+11$ & $4.0 \mathrm{e}+08$ & $7.2 \mathrm{e}+04$ & $2.0 \mathrm{e}+05$ & 0.073 \\
\hline
\end{tabular}

Table 2: Comparison of Simulation Result

In Table 2, SSE denotes the sum of squares due to error, MSE is the mean squared error and MAE is the mean absolute error.

As to the online prediction, time is also an important index for consideration. The prediction time of SVM and grey SVM prediction scheme are at the same level; but they have an obviously advantage than other methods on time. The accuracy of Grey SVM is better than the conventional SVM network which is based on RMSE and the accuracy is also better than other neural networks. The result also shows that Grey model is fit for some other neural networks; however, Grey SVM still has advantages against other methods both in terms of time and accuracy which are important for the online prediction.

\section{Conclusion}

SVM is a network which is suitable to represent the nonlinear mapping of system, and grey predict model can eliminate part of random error and make the result more accurate. In this study, a grey SVM is constructed in combination with both methods and the achieved predictive model is applied to the online ship roll motion prediction. Simulation result shows that the proposed grey prediction system generates roll angle prediction with higher accuracy than the conventional SVM, which has demonstrated the effectiveness and applicability of the proposed system.

\section{References}

[1] T. I. Fossen. Handbook of Marine Craft Hydrodynamics and Motion Control, John Wiley \& Sons Ltd., USA, pp, 296-307(2011)

[2] T. I. Fossen Guidance and Control of Ocean Vehicles, John Wiley \& Sons Ltd., USA, pp, 101110 (1994)

[3] F. Xu, Z. J. Zou, J. C. Yin. On-line Modeling of Ship Maneuvering Motion based on Support Vector Machine. Journal of Ship Machanics,16(3): 218-225(2012)

[4] J. L. Deng, Grey System Fundamental Method, Huazhong University of Science and Technology Press, Wuhan, China, pp, 27-40 (1987) (In Chinese)

[5] S. L. Ou. Forecasting agricultural output with and improved grey forecasting model based on the genetic algorithm, Comput. Electron. Agri. 85(1),:33-39(2012)

[6] C. H. Wang, L.C. Hsu. Using genetic algorithms grey theory to forecast high technology industrial output, Appl. Math. Comput. 195(1), pp:256-263(2008) 
[7] Y. H. Lin, C. C. Chiu, P. C. Lee, Y. J. Lin. Applying fuzzy grey modification model on inflow forecasting, Eng. Appl. Artif. Intell. 25(4), pp:734-743(2012)

[8] L. S. Wei, M. R. Fei, H. S. Hu. Modeling and stability analysis of grey-fuzzy predictive control, Neurocomputing 72(1-3),:197-202(2008)

[9] C. Cortes, V. Vapnik Support-vector networks. Machine learning, v20(3):273-297(1995)

[10] J. Deng. Grey Prediction and Decision, Huazhong University of Science and Technology, Wuhan(2002) 